\title{
Contribution to the search of binaries among Am stars
}

\section{HD 81976 and HD 98880, double-lined spectroscopic binaries}

\author{
J.-M. Carquillat, N. Ginestet, and J.-L. Prieur
}

UMR 5572 d'Astrophysique, Observatoire Midi-Pyrénées - Centre National de la Recherche Scientifique, 14 avenue Édouard Belin, 31400 Toulouse, France

Received 22 November 2000 / Accepted 15 January 2001

\begin{abstract}
We present the results of the observations of two Am stars of eighth magnitude, the double-lined spectroscopic binaries HD 81976 and HD 98880, carried out with the CORAVEL instrument at the Observatoire de Haute-Provence in order to determine their orbital elements. We found 1) for HD 81976: $P=5.655750$ days, $T=2449785.941$ HJD $, \omega=341.4^{\circ}, e=0.061, K_{1}=61.68 \mathrm{~km} \mathrm{~s}^{-1}, K_{2}=63.84 \mathrm{~km} \mathrm{~s}^{-1}, V_{0}=19.85 \mathrm{~km} \mathrm{~s}^{-1}$, $a_{1} \sin i=4.788 \mathrm{Gm}, a_{2} \sin i=4.956 \mathrm{Gm}, M_{1} \sin ^{3} i=0.5875 M_{\odot}, M_{2} \sin ^{3} i=0.5676 M_{\odot}$, and 2) for HD 98880 : $P=14.20783$ days, $T_{0}=2448682.883$ HJD (ascending node), $e=0 ., K_{1}=42.47 \mathrm{~km} \mathrm{~s}^{-1}, K_{2}=49.16 \mathrm{~km} \mathrm{~s}{ }^{-1}$, $V_{0}=2.40 \mathrm{~km} \mathrm{~s}^{-1}, a_{1} \sin i=8.298 \mathrm{Gm}, a_{2} \sin i=9.604 \mathrm{Gm}, M_{1} \sin ^{3} i=0.6091 M_{\odot}, M_{2} \sin ^{3} i=0.5262 M_{\odot}$. The first of these two systems, HD 81976, is formed by two quasi-identical stars, and the Hipparcos data $\left(M_{V}, B-V\right)$ are consistent with late A stars in effective temperature; it is likely that the components rotate synchronised with the orbital motion. A third body may be present in this system since (i) the orbit has a significant eccentricity despite its short period and (ii) the systemic velocity $V_{0}$ shows a possible drift. For the second system, HD 98880, we give $\Delta m_{B}=1.25$ and we propose a simple model based upon Strömgren photometric indices and the HR theoretical diagram of Schaller et al (1992) in addition to orbital parameters and Hipparcos data: $T_{\text {eff }}=7000 \mathrm{~K}$, $\log _{10} g=4.0, M_{1}=1.9 M_{\odot}, M_{2}=1.6 M_{\odot}, \log _{10}($ age $)=9.12$. The components do not rotate synchronously contrary to HD 81976. Both binaries appear to be detached systems without possibility of eclipses.
\end{abstract}

Key words. stars: individual: HD 81976, HD 98880 - binaries: spectroscopic - stars: fundamental parameters

\section{Introduction}

HD 81976 (HIP 46588) and HD 98880 (HIP 55565) belong to our sample of Am-type stars that we have been observing with the CORAVEL instrument of "Observatoire de Haute-Provence" (OHP, Haute-Provence Observatory) in order to contribute to the study of the frequency of binaries in this stellar family (cf. Ginestet \& Carquillat 1998, Paper I; North et al. 1998).

The first of these two stars, HD 81976, was reported as Am in a paper by Bidelman (1988), in his "Miscellaneous spectroscopic notes", whereas the second, HD 98880, was included in the "Third catalogue of Am stars with known spectral types" (Hauck 1986), which was the main source used for constructing our sample. Note that the Am type was attributed to HD 98880 by Abt (1984) during his spectroscopic investigation of a sample of possible peculiar

Send offprint requests to: J.-M. Carquillat,

e-mail: jean-michel.carquillat@obs-mip.fr

* Based on observations made at the Haute-Provence Observatory, France. stars which had peculiar indices in Strömgren photometry (Olsen 1980). Concerning their binarity, only HD 81976 had already been reported in the past as having a doublelined spectrum (Adam 1915) from observations carried out at Mount Wilson, but to our knowledge, no spectroscopic orbit had ever been obtained.

Our observations lead to the determination of the orbital parameters for these two double-lined spectroscopic binaries.

\section{Radial velocity observations and determination of the orbital parameters}

The radial velocities $(R V)$ have been obtained with the CORAVEL spectro-velocimeter (Baranne et al. 1979) mounted at the Cassegrain focus of the 1-m Swiss telescope of OHP. The measurements were processed and included into the data base of Geneva Observatory by S. Udry. The $R V$ are in the IAU standard system.

HD 81976 was observed during the years 1995 to 1998. Forty one observations have been performed (Table 1), 
Table 1. Radial velocities of HD 81976. $\mathrm{RV}_{1},(\mathrm{O}-\mathrm{C})_{1}$ : radial velocities and residuals for the primary; $\mathrm{RV}_{2},(\mathrm{O}-\mathrm{C})_{2}$ : same data for the secondary. The phases are from the periastron passage

\begin{tabular}{|c|c|c|c|c|c|c|c|}
\hline $\mathrm{N}^{\circ}$ & Date & $\begin{array}{c}\text { HJD } \\
2400000+\end{array}$ & Phase & $\begin{array}{c}\mathrm{RV}_{1} \\
\mathrm{~km} \mathrm{~s}^{-1} \\
\end{array}$ & $\begin{array}{c}(\mathrm{O}-\mathrm{C})_{1} \\
\mathrm{~km} \mathrm{~s}^{-1}\end{array}$ & $\begin{array}{l}\mathrm{RV}_{2} \\
\mathrm{~km} \mathrm{~s}^{-1}\end{array}$ & $\begin{array}{c}(\mathrm{O}-\mathrm{C})_{2} \\
\mathrm{~km} \mathrm{~s}^{-1}\end{array}$ \\
\hline 1 & 07.03 .1995 & 49783.539 & 0.575 & -36.9 & +1.1 & +82.4 & +2.7 \\
\hline 2 & 07. & 49784.391 & 0.726 & -9.8 & +1.4 & +52.0 & +0.0 \\
\hline 3 & 08. & 49785.309 & 0.888 & +50.9 & +0.1 & -11.5 & +0.6 \\
\hline 4 & 08. & 49785.457 & 0.914 & +61.1 & +0.8 & -20.8 & +1.2 \\
\hline 5 & 09. & 49785.688 & 0.955 & +72.3 & -0.5 & & \\
\hline 6 & 09. & 49786.289 & 0.061 & +85.4 & +0.7 & -47.3 & -0.0 \\
\hline 7 & 09. & 49786.457 & 0.091 & +82.3 & +0.3 & -44.4 & +0.1 \\
\hline 8 & 10. & 49787.305 & 0.241 & +40.4 & +1.2 & +0.7 & +0.9 \\
\hline 9 & 10. & 49787.426 & 0.262 & +32.2 & +1.0 & +10.4 & +2.3 \\
\hline 10 & 11.02 .1996 & 50124.590 & 0.877 & +47.7 & +1.3 & -7.8 & -0.2 \\
\hline 11 & 12. & 50125.527 & 0.043 & +85.3 & +0.2 & -47.2 & +0.5 \\
\hline 12 & 13. & 50126.590 & 0.230 & +44.3 & +1.1 & -4.4 & -0.1 \\
\hline 13 & 14. & 50127.578 & 0.405 & -15.0 & +2.0 & +57.3 & -0.7 \\
\hline 14 & 19.04 .1996 & 50192.512 & 0.886 & +50.2 & +0.2 & -10.4 & +1.0 \\
\hline 15 & 19. & 50193.438 & 0.050 & +85.1 & +0.0 & -49.2 & -1.5 \\
\hline 16 & 20. & 50194.434 & 0.226 & +45.4 & +0.6 & -6.0 & -0.0 \\
\hline 17 & 27.11 .1996 & 50414.715 & 0.174 & +62.9 & +0.3 & -23.8 & +0.6 \\
\hline 18 & 28. & 50415.660 & 0.341 & +0.9 & -1.5 & +35.8 & -2.1 \\
\hline 19 & 29. & 50416.551 & 0.499 & -34.2 & +0.7 & +75.9 & -0.6 \\
\hline 20 & 01.12 .1996 & 50418.574 & 0.857 & +38.8 & +0.5 & -0.1 & -0.8 \\
\hline 21 & 01. & 50418.742 & 0.886 & +50.0 & -0.1 & -11.9 & -0.5 \\
\hline 22 & 01. & 50419.484 & 0.017 & +83.2 & -0.6 & -47.3 & -0.9 \\
\hline 23 & 02. & 50419.586 & 0.035 & +84.6 & -0.3 & & \\
\hline 24 & 03. & 50420.512 & 0.199 & +53.3 & -1.1 & -15.7 & +0.2 \\
\hline 25 & 03. & 50420.621 & 0.218 & +46.4 & -1.1 & -10.9 & -2.1 \\
\hline 26 & 04. & 50421.570 & 0.386 & & & +53.7 & +1.1 \\
\hline 27 & 29.01 .1997 & 50478.348 & 0.425 & -23.2 & -1.2 & +60.7 & -2.5 \\
\hline 28 & 29. & 50478.488 & 0.450 & -27.6 & -0.2 & +68.0 & -0.7 \\
\hline 29 & 30. & 50478.742 & 0.495 & & & +75.0 & -1.1 \\
\hline 30 & 30. & 50479.320 & 0.597 & -38.5 & -1.7 & +78.1 & -0.4 \\
\hline 31 & 31. & 50479.527 & 0.634 & -32.8 & -0.1 & +73.5 & -0.7 \\
\hline 32 & 31. & 50480.375 & 0.784 & +11.8 & +2.7 & +33.2 & +2.2 \\
\hline 33 & 01.02 .1997 & 50480.734 & 0.847 & +32.6 & -1.9 & +3.0 & -1.6 \\
\hline 34 & 01. & 50481.336 & 0.954 & +71.4 & -0.9 & -35.6 & -1.1 \\
\hline 35 & 02. & 50481.586 & 0.998 & +82.2 & +0.7 & -43.4 & +0.6 \\
\hline 36 & 02. & 50482.371 & 0.137 & & & -36.1 & -0.7 \\
\hline 37 & 15.06 .1997 & 50615.352 & 0.649 & -29.8 & +0.4 & & \\
\hline 38 & 15. & 50615.363 & 0.651 & & & +70.4 & -0.9 \\
\hline 39 & 24.10 .1997 & 50745.719 & 0.699 & -18.8 & +0.1 & +59.2 & -0.7 \\
\hline 40 & 23.01 .1998 & 50836.543 & 0.758 & -0.9 & -0.5 & +40.8 & +0.0 \\
\hline 41 & 25. & 50838.563 & 0.115 & +78.1 & +0.1 & -41.7 & -1.4 \\
\hline
\end{tabular}




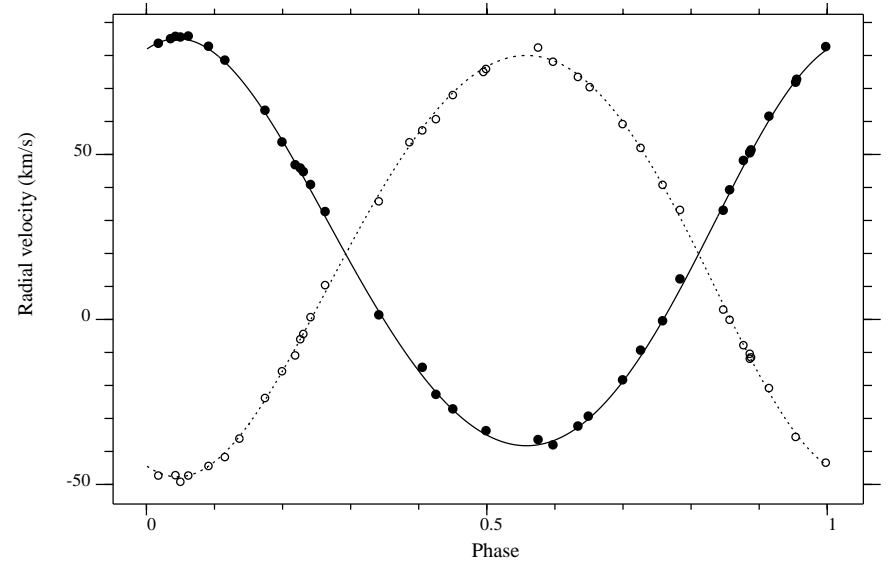

Fig. 1. Radial velocity curves for HD 81976. Filled circles: main component, open circles: secondary component. The origin for phases corresponds to the periastron passage

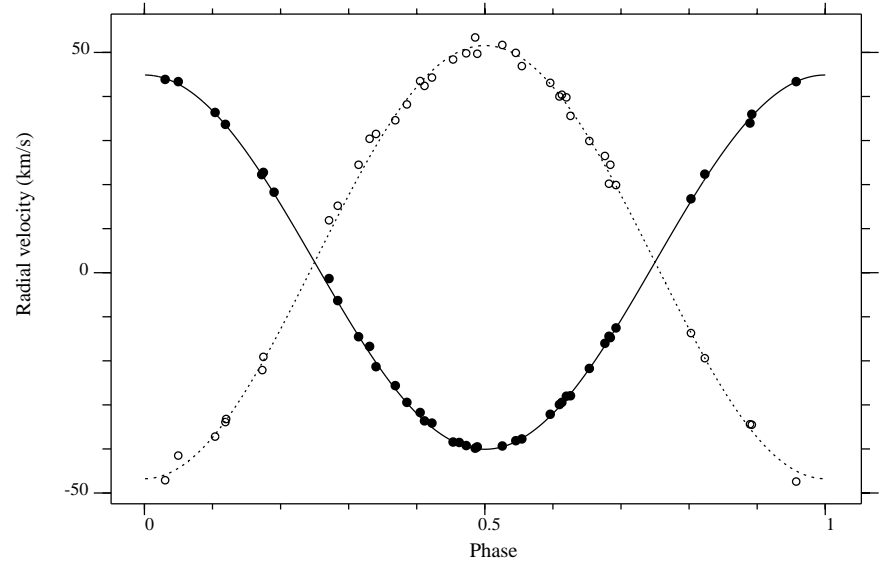

Fig. 2. Radial velocity curves for HD 98880. Filled circles: main component, open circles: secondary component. The origin for phases corresponds to the ascending node passage

which cover 186 orbital cycles. The mean internal standard error of the $R V$ measurements is $0.9 \mathrm{~km} \mathrm{~s}^{-1}$ for the primary component and $1.0 \mathrm{~km} \mathrm{~s}^{-1}$ for the secondary. The correlation dips (i.e. the profiles of the crosscorrelation function of the absorption lines as measured by CORAVEL) of both components are very similar.

HD 98880 was observed from 1992 to 1998. Forty two observations were performed (Table 2), which cover 162 orbital cycles. Contrary to the previous case, the correlation dips of both components are rather different, the profile of the secondary component being much shallower. The mean internal standard error for the $R V$ are respectively $0.5 \mathrm{~km} \mathrm{~s}^{-1}$ and $1.1 \mathrm{~km} \mathrm{~s}^{-1}$ for the main and secondary components.

We have computed the orbital elements with our BS2 program (Nadal et al. 1979) which was improved by one of us (J.-L. P.). Taking into account the mean standard errors of the measurements, the $R V$ of each component were weighted as follows: for HD 81976, 1.0 for the primary component and 0.8 for the secondary, and for HD 98880 , 1.0 for the primary and $1 / 4$ for the secondary.
The orbital elements that we obtained are given in Table 3 and the $R V$ curves in Figs. 1 and 2. Note the small eccentricity of 0.06 for HD 81976, which has nevertheless the shortest period. On the contrary, for the second system, the eccentricity had to be set to zero for a good fit.

\section{Discussion}

\section{1. $H D 81976$}

As mentioned in the previous section, the correlation dips of both components are quite similar, which indicates that the two stars must have a similar type (Am in that case). This is supported by the value of the mass ratio $r=0.97$, very close to unity. Assuming that these stars have a mass of the order of $2 M_{\odot}$, the value obtained for $M_{1} \sin ^{3} i=0.5875 M_{\odot}$ would lead to $i=42^{\circ}$, which implies a quasi-certain impossibility of eclipse occurrence. With the same assumption, we would have $a=a_{1}+a_{2}=14.66 \mathrm{Gm}=21 R_{\odot}$, and the system would appear as detached.

\subsubsection{Comparison with the Hipparcos measurements}

For HD $81976=$ HIP 46588, the Hipparcos catalogue (ESA 1997) gives: $V=8.17, \pi=5.65 \pm 1.18$ mas, $B-V=0.285, V-I=0.32$.

From the value for the trigonometric parallax, the distance of this system is about $180 \mathrm{pc}$, and the absolute $V$ magnitude can be estimated at $1.93 \pm 0.46$, assuming negligible interstellar absorption. If we assume both components have the same luminosity, we obtain $M_{V}=2.68 \pm 0.46$ for each component. This value is compatible with Am-type stars as shown by North et al. (1997), who obtained absolute $V$ magnitudes in the range +0.3 and +2.8 for a sample of 96 Am stars observed with the Hipparcos satellite.

Note also that the color indices as measured by Hipparcos are consistent with a late A-type star, about A7-A8 (Schmidt-Kaler 1982). Such a spectral type should correspond to the strength of hydrogen lines and the effective temperature of the star (Bidelman does not give any detail for his classification but only the result: "Am"). This is also consistent with the dA7 classification given in the Mount Wilson catalogue (Wilson 1953), since such a classification was done at a time when the Am stars were not yet known as a family of its own.

\subsubsection{Rotation-revolution synchronism}

Since the orbital period is rather short, a synchronism can be expected between the axial rotation and the orbital revolution of the components. The test proposed by Kitamura \& Kondo (1978) consists of calculating the radii that the two stars would have if synchronism was effective. If the values found for these radii are compatible with those expected for stars of similar spectral type, 
Table 2. Radial velocities of HD 98880. $\mathrm{RV}_{1},(\mathrm{O}-\mathrm{C})_{1}$ : radial velocities and residuals for the primary; $\mathrm{RV}_{2},(\mathrm{O}-\mathrm{C})_{2}$ : same data for the secondary. The phases are from the ascending node passage

\begin{tabular}{|c|c|c|c|c|c|c|c|}
\hline $\mathrm{N}^{\circ}$ & Date & $\begin{array}{c}\text { HJD } \\
2400000+\end{array}$ & Phase & $\begin{array}{l}\mathrm{RV}_{1} \\
\mathrm{~km} \mathrm{~s}^{-1}\end{array}$ & $\begin{array}{c}(\mathrm{O}-\mathrm{C})_{1} \\
\mathrm{~km} \mathrm{~s}^{-1}\end{array}$ & $\begin{array}{l}\mathrm{RV}_{2} \\
\mathrm{~km} \mathrm{~s}^{-1}\end{array}$ & $\begin{array}{c}(\mathrm{O}-\mathrm{C})_{2} \\
\mathrm{~km} \mathrm{~s}^{-1}\end{array}$ \\
\hline 1 & 23.02 .1992 & 48675.578 & 0.486 & -40.2 & -0.3 & +53.4 & +2.0 \\
\hline 2 & 23. & 48676.426 & 0.546 & -38.5 & -0.2 & +49.9 & +0.3 \\
\hline 3 & 11.12 .1992 & 48967.746 & 0.050 & +43.0 & +0.2 & -41.5 & +2.9 \\
\hline 4 & 12. & 48968.730 & 0.119 & +33.3 & -0.2 & -33.9 & -0.3 \\
\hline 5 & 12. & 48968.746 & 0.120 & & & -33.2 & +0.2 \\
\hline 6 & 13. & 48969.746 & 0.191 & +17.9 & -0.0 & & \\
\hline 7 & 13.01 .1993 & 49000.691 & 0.369 & -26.0 & +0.4 & +34.6 & -1.1 \\
\hline 8 & 02.06 .1993 & 49141.387 & 0.271 & -1.7 & +1.5 & +11.9 & +3.0 \\
\hline 9 & 03. & 49142.367 & 0.340 & -21.7 & \begin{tabular}{|l|l}
-1.3 \\
\end{tabular} & +31.5 & +2.7 \\
\hline 10 & 04. & 49143.379 & 0.411 & -34.0 & -0.3 & +42.4 & -1.7 \\
\hline 11 & 05. & 49144.480 & 0.489 & -39.9 & +0.1 & +49.7 & -1.7 \\
\hline 12 & 06. & 49145.410 & 0.554 & -38.1 & -0.5 & +46.9 & -1.8 \\
\hline 13 & 07. & 49146.426 & 0.626 & -28.3 & -0.8 & +35.6 & -1.4 \\
\hline 14 & 08. & 49147.375 & 0.693 & -12.9 & -0.3 & +19.9 & +0.2 \\
\hline 15 & 26.11 .1993 & 49317.723 & 0.682 & -14.8 & +0.3 & +20.2 & -2.5 \\
\hline 16 & 28. & 49319.723 & 0.823 & +22.0 & +0.8 & -19.4 & +0.0 \\
\hline 17 & 29. & 49320.703 & 0.892 & +35.6 & +0.1 & -34.5 & +1.4 \\
\hline 18 & 02.12 .1993 & 49323.711 & 0.104 & +36.0 & -0.1 & -37.2 & -0.5 \\
\hline 19 & 03. & 49324.719 & 0.175 & +22.4 & +0.7 & -19.1 & +0.9 \\
\hline 20 & 16.03 .1994 & 49427.691 & 0.422 & -34.5 & +0.6 & +44.3 & -1.5 \\
\hline 21 & 16. & 49428.406 & 0.473 & -39.6 & -0.2 & +49.8 & -1.0 \\
\hline 22 & 18 & 49430.402 & 0.613 & -29.8 & -0.0 & +40.4 & +0.8 \\
\hline 23 & 05.03 .1995 & 49781.586 & 0.331 & -17.1 & +1.1 & +30.4 & +4.1 \\
\hline 24 & 06. & 49782.641 & 0.405 & -32.1 & +0.6 & +43.5 & +0.4 \\
\hline 25 & 09. & 49785.547 & 0.610 & -30.3 & +0.1 & +40.0 & -0.4 \\
\hline 26 & 09. & 49786.496 & 0.676 & -16.4 & +0.1 & +26.5 & +2.2 \\
\hline 27 & 13.02 .1996 & 50126.680 & 0.620 & -28.4 & +0.2 & +39.8 & +1.5 \\
\hline 28 & 14 & 50127.598 & 0.684 & -15.1 & -0.5 & +24.5 & +2.4 \\
\hline 29 & 19.04 .1996 & 50193.383 & 0.315 & -14.9 & -0.5 & +24.5 & +2.7 \\
\hline 30 & 20. & 50194.391 & 0.386 & -29.8 & -0.2 & +38.2 & -1.2 \\
\hline 31 & 21. & 50195.484 & 0.463 & -38.9 & +0.0 & & \\
\hline 32 & 27.11 .1996 & 50414.668 & 0.889 & +33.6 & -1.4 & -34.4 & +1.0 \\
\hline 33 & 28 & 50415.633 & 0.957 & +43.0 & -0.4 & -47.4 & -2.4 \\
\hline 34 & 29. & 50416.672 & 0.031 & +43.5 & -0.6 & -47.1 & -1.2 \\
\hline 35 & 01.12 .1996 & 50418.688 & 0.172 & +21.9 & -0.4 & & \\
\hline 36 & 01 & 50418.699 & 0.173 & & & -22.1 & -1.7 \\
\hline 37 & 31.01 .1997 & 50479.512 & 0.453 & -38.8 & -0.5 & +48.4 & -1.1 \\
\hline 38 & 01.02 .1997 & 50480.539 & 0.526 & -39.7 & -0.2 & +51.7 & +0.8 \\
\hline 39 & 02. & 50481.539 & 0.596 & -32.5 & +0.1 & +43.1 & +0.2 \\
\hline 40 & 24.01 .1998 & 50837.551 & 0.654 & -22.1 & -0.3 & +29.9 & -0.5 \\
\hline 41 & 26. & 50839.672 & 0.803 & +16.4 & +0.2 & -13.7 & -0.1 \\
\hline 42 & 09.06 .1998 & 50974.379 & 0.284 & -6.7 & -0.1 & +15.2 & +2.4 \\
\hline
\end{tabular}


Table 3. Orbital elements and their standard errors for the systems HD 81976 and HD 98880

\begin{tabular}{|l|c|l|l|l|}
\hline \multicolumn{1}{|c|}{ Element } & HD 81976 & Std error & HD 98880 & Std error \\
\hline$P$ (days) & 5.655750 & 0.000050 & 14.20783 & 0.00018 \\
\hline$T_{0}{ }^{*}(\mathrm{HJD})$ & 2449785.941 & 0.045 & 2448682.883 & 0.015 \\
\hline$\omega$ (degrees) & 341.4 & 3.0 & & \\
\hline$e$ & 0.061 & 0.003 & 0 (fixed) & \\
\hline$K_{I}\left(\mathrm{~km} \mathrm{~s}^{-1}\right)$ & 61.68 & 0.26 & 42.47 & 0.17 \\
\hline$K_{2}\left(\mathrm{~km} \mathrm{~s}^{-1}\right)$ & 63.84 & 0.28 & 49.16 & 0.33 \\
\hline$V_{0}\left(\mathrm{~km} \mathrm{~s}^{-1}\right)$ & 19.85 & 0.14 & 2.40 & 0.11 \\
\hline$a_{1} \sin i(\mathrm{Gm})$ & 4.788 & 0.020 & 8.298 & 0.033 \\
\hline$a_{2} \sin i(\mathrm{Gm})$ & 4.956 & 0.022 & 9.604 & 0.064 \\
\hline$M_{1} \sin ^{3} i\left(\mathrm{M}_{\odot}\right)$ & 0.5875 & 0.0057 & 0.6091 & 0.0088 \\
\hline$M_{2} \sin ^{3} i\left(\mathrm{M}_{\odot}\right)$ & 0.5676 & 0.0054 & 0.5262 & 0.0056 \\
\hline$\sigma_{1}(\mathrm{O}-\mathrm{C}) \mathrm{km} \mathrm{s}^{-1}$ & 1.0 & & 0.55 & \\
\hline$\sigma_{2}(\mathrm{O}-\mathrm{C}) \mathrm{km} \mathrm{s}^{-1}$ & 1.2 & & 1.7 & \\
\hline
\end{tabular}

* $T_{0}$ : periastron passage for HD 81976 ; ascending node passage for HD 98880

one can deduce that the hypothesis of synchronism is plausible. Actually, the equatorial velocity for synchronism is $V_{\mathrm{s}}=50.6\left(R / R_{\odot}\right) / P$.

In our case, $V_{\mathrm{s}}$ can be estimated from $v \cdot \sin i$ as deduced from the profile of the correlation dips (method described in Benz \& Mayor 1981), which is $14.5 \pm 0.5 \mathrm{~km} \mathrm{~s}^{-1}$. Assuming $i=42^{\circ}$ and the coplanarity between the equatorial and the orbital planes, one finds $V_{\mathrm{s}}=21.8 \mathrm{~km} \mathrm{~s}^{-1}$ and hence $R=2.4 R_{\odot}$. This value is quite compatible with those deduced from the study of similar systems showing eclipses (Kitamura \& Kondo 1978). We can then conclude that the hypothesis of synchronism is relevant for HD 81976.

\subsubsection{Hypothesis of a third body}

In Sect. 2, we noticed that the eccentricity of the orbit of HD 81976 was not zero and that the other system studied here has a period nearly 2.5 times larger with a circular orbit. The existence of a non-circular orbit for a system with a period as short as 5.6 days is surprising. Actually, the study of a sample of Am stars belonging to the Hyades and Praesepe clusters by Debernardi et al. (2000) shows that orbits are quasi circular for $P<10$ days, and the authors note that this is in agreement with the theoretical work of Zahn \& Bouchet (1989).

On the other hand, when plotting the residuals $(\mathrm{O}-\mathrm{C})$ of Table 1 versus time (Fig. 3), one notes they seem to decrease with time, which shows a possible drift of the velocity $V_{0}$ of the system's gravity center.

These two points lead us to suggest the presence of a third body in this system. We have already formulated such an hypothesis for the Am-type system HD 66068/9 (Carquillat et al. 1994), which also showed a $V_{0}$ drift and

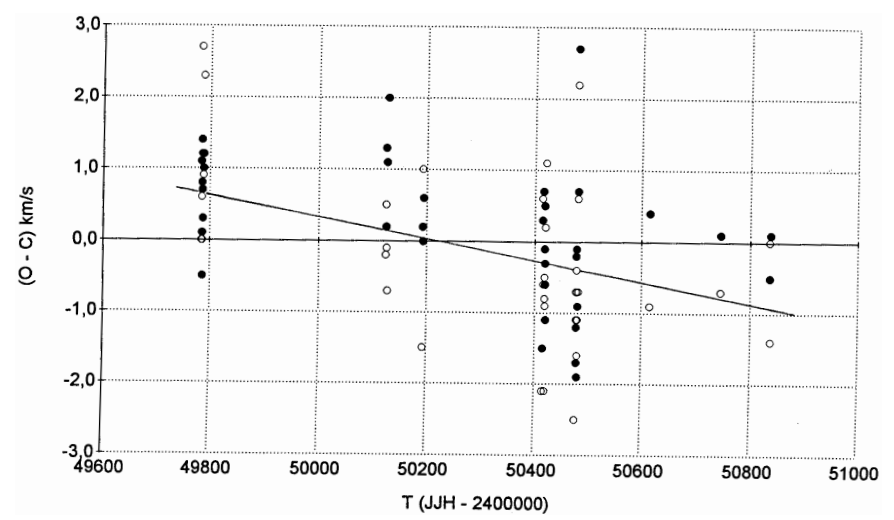

Fig. 3. Variation of the residuals $(\mathrm{O}-\mathrm{C})$ versus time for HD 81976. Filled circles: primary component, open circles: secondary component. The straight line with a negative slope corresponds to the least square fit

exhibited an abnormally high eccentricity for a period close to 8 days.

\section{2. $H D 98880$}

For this binary, the mass ratio of 0.86 is an indication that the two components have a similar type, but contrary to the previous case, the depths of the correlation dips are significantly different, which implies that their luminosities are also different. Actually, the magnitude difference of the two components can be inferred from the value of the ratio of the integrated aeras of the dips (Lucke \& Mayor 1980). For HD 98880, we obtain $\Delta m_{B}=1.25 \pm 0.1 \mathrm{mag}$. 


\subsubsection{Physical parameters and evolution state of the system}

The Hipparcos parallax, $\pi=4.25 \pm 0.91$ mas (i.e. $d=235 \mathrm{pc}$ ), combined with the apparent magnitude $V=8.06$ leads to a global absolute magnitude of $M_{V}=1.20 \pm 0.46$ for the system, if we neglect the interstellar absorption. If we assume that the effective temperatures of the two components are similar $\left(T_{1} \approx T_{2}\right)$, $\Delta m_{V} \approx \Delta m_{B}$, and if we use the value of $\Delta m_{B}=1.25 \mathrm{mag}$ found previously, we obtain the absolute $V$ magnitudes of both components: $M_{V 1}=1.5 \pm 0.5$ and $M_{V 2}=2.75 \pm 0.5$.

For HD 98880, Olsen (1983) gives the following Strömgren photometry indices $V=8.063, b-y=0.228$, $m_{1}=0.243, c_{1}=0.642$; the $\beta$ index was not given by Olsen. The calibration by Crawford (1979) leads to $\beta=2.72$ and $\delta m_{1}=-0.066$ (blanketing parameter). These data allow us to estimate the effective temperature and the metallicity. The effective temperature, as well as the surface gravity, can be obtained with the theoretical grids computed by Moon \& Dworetsky (1985). In this case we find: $T \approx 7000 \mathrm{~K}$ and $\log _{10} g \approx 4$.0. This value for $T$ seems both compatible with the classification made by Abt (1984): A4-F3-F5 respectively for the $\mathrm{K}$ line, the Balmer lines and the metallic lines, and with the color index $B-V=0.39$ reported in the Hipparcos catalogue (ESA, 1997). As for the metallicity, it can be estimated from the relation $\delta m_{1}$ versus $[\mathrm{Fe} / \mathrm{H}]$ established by Crawford (1975). In this case, $[\mathrm{Fe} / \mathrm{H}] \approx 0.8 \mathrm{dex}$, which is a good signature of Am character.

On the other hand, if we refer to the theoretical HR diagram computed by Schaller et al. (1992) for solar metallicity stars (Fig. 4), the masses of the two components would be about $1.9 M_{\odot}$ and $1.6 M_{\odot}$, whose ratio is close to that of the $R V$ curve amplitudes. To locate each component in this diagram, we applied a correction of $-0.1 \mathrm{mag}$ to the absolute magnitude (i.e., bolometric correction for $T=7000 \mathrm{~K})$ to derive $M_{\mathrm{bol}}$ and the luminosity $L$.

We can then propose the following (rough) model: the system HD 98880 is about $1.310^{9}$ years old and constituted by two Am stars with effective temperature close to $7000 \mathrm{~K}$; the primary $\left(1.9 M_{\odot}\right)$ would be already a rather evolved main sequence star whereas the secondary $\left(1.6 M_{\odot}\right)$ would be only about $1 \mathrm{mag}$ above the zero-age main sequence. The corresponding theoretical radii would be $3.3 R_{\odot}$ for the primary and $1.9 R_{\odot}$ for the companion.

\subsubsection{System dimensions, rotation-revolution synchronism}

The value of $1.9 M_{\odot}$ found for the mass of the primary would imply $i=43^{\circ}, a=26.16 \mathrm{Gm}=37.6 R_{\odot}$. Hence, like HD 81976, HD 98880 is a detached system without the possibility of eclipses.

The values of $v \cdot \sin i$ deduced from the correlation dip profiles are $10.6 \pm 0.2 \mathrm{~km} \mathrm{~s}^{-1}$ for the primary and $9.2 \pm 0.7 \mathrm{~km} \mathrm{~s}^{-1}$ for the secondary. We applied the Kiramura \& Kondo test with these values and $i=43^{\circ}$.

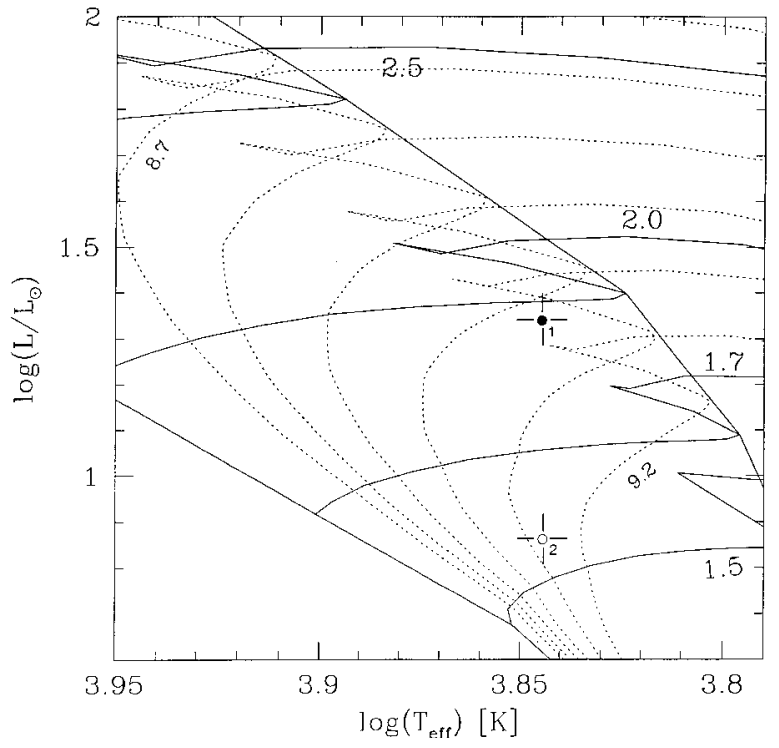

Fig. 4. Estimated location of the primary (1) and secondary (2) components for the HD 98880 system in the HR diagram with theoretical evolution curves for solar metallicity stars computed by Schaller et al. (1992)

Assuming the coplanarity between the orbital and equatorial planes, the rotation-revolution synchronism would require radii of respectively $4.35 R_{\odot}$ and $3.8 R_{\odot}$ for the primary and secondary. These values are significantly larger than those $\left(3.3 R_{\odot}\right.$ and $\left.1.9 R_{\odot}\right)$ corresponding to the model described above. We conclude that, unlike HD 81976, the HD 98880 system has not yet reached the synchronism state, which is not surprising in view of its much longer orbital period.

Acknowledgements. We are indebted to Michel Mayor, Director of Geneva Observatory, for giving us observing time with CORAVEL and to Stéphane Udry for processing the radial velocity measurements. For bibliographic references, this work made use of the SIMBAD database, operated at the "Centre de Données astronomiques de Strasbourg" (France).

\section{References}

Abt, H. A. 1984, ApJ, 285, 247

Adams, W. S. 1915, PASP, 27, 132

Baranne, A., Mayor, M., \& Poncet, J. L. 1979, Vistas Astron., 23, 279

Benz, W., \& Mayor, M. 1981, A\&A, 93, 235.

Bidelman, W. P. 1988, PASP, 100, 1084

Carquillat, J. M., Ginestet, N., Duquennoy, A., \& Pédoussaut, A. 1994, A\&AS, 106, 597

Crawford, D. L. 1975, AJ, 80, 955

Crawford, D. L. 1979, AJ, 84, 1858

Debernardi, Y. Mermilliod, J.-C., Carquillat, J.-M., \& Ginestet, N. 2000, A\&A, 354, 881

ESA, 1997, The Hipparcos and Tycho Catalogues, ESA SP-1200

Ginestet, N., \& Carquillat, J.-M. 1998, A\&AS, 130, 415 (Paper I)

Hauck, B. 1986, A\&AS, 64, 21 
Kitamura, M., \& Kondo, M. 1978, Ap\&SS, 56, 341

Lucke, P. B., \& Mayor, M. 1980, A\&A, 92, 182

Moon, T. T., \& Dworetsky, M. M. 1985, MNRAS, 217, 305

Nadal, R., Ginestet, N., Carquillat, J.-M., \& Pédoussaut, A. 1979, A\&AS, 35, 203

North, P., Jaschek, C., Hauck, B., et al. 1997, Proc. ESA Symp. Hipparcos-Venice'97, ESA SP-402, 239

North, P., Ginestet, N., Carquillat, J.-M., Carrier, F., \& Udry, S. 1998, Contrib. Astron. Obs. Skalnaté Pleso, 27, 179

Olsen, E. H. 1980, A\&AS, 39, 205
Olsen, E. H. 1983, A\&AS, 54, 55

Schaller, G., Schaerer, D., Meynet, G., \& Maeder, A. 1992, A\&AS, 96, 269

Schmidt-Kaler, Th. 1982, in Landolt-Börnstein, New Series, Gr. 6, vol. 2-B (Springer-Verlag: Berlin, Heidelberg, NewYork), 1

Wilson, R. E. 1953, General Catalogue of Stellar Radial Velocities (Carnegie Institution Washington Pub. 601)

Zahn, J.-P., \& Bouchet, L. 1989, A\&A, 223, 112 